\section{Parkinson's yield sign}

\section{By Steve Edelson, Executive Editor}

A paper by Korean researchers published in the Proceedings of the National Academy of Sciences describes the highest-ever yield of dopamine-producing neurons from human embryonic stem cells. But companies polled by SciBX think that stem cell yield is just one of many hurdles in using human embryonic stem cells to treat Parkinson's disease.

The hallmark of Parkinson's disease (PD) is the loss of dopaminergic neurons (DA neurons) that originate in the substantia nigra and project into the striatum. Thus, marketed PD drugs either agonize dopamine receptors or introduce the neurotransmitter itself into the system. But patients inevitably become desensitized to the exogenous dopamine, and neither class of drugs modifies the underlying disease.

In the PNAS article, researchers at the Stem Cell Research Center in Seoul and colleagues cultured human embryonic stem cell (hESC) colonies on bacterial dishes and selected for neural precursors. ${ }^{1}$ The precursors were expanded and those that formed neural clumps were isolated.

After 10 days, the clumps formed spherical neural masses (SNMs), which the researchers wrote was "the unique feature of our protocol. The SNMs can be expanded for long periods without losing their differentiation capability and be coaxed into DA neurons efficiently" within about two weeks.

The DA neurons were used in a rat model of PD and produced "clear behavioral recovery," Dong-Wook Kim told SciBX. Kim is director of the research center and an author on the paper.

Kim added that the rats had no teratoma formation and that the stem cells were differentiated without coculturing using a feeder layer, which eliminates the risk of contamination. The method generates "dopamine neurons that are much closer to clinical application than any other methods," he said.

Kim did note that many hurdles remain before the dopamine neurons are ready for the clinic, including obtaining further increases in yield and developing techniques to remove cells that do not differentiate.

"The hypothesis of dopamine treatment is beyond questionreplace dopamine in the striatum and you'll treat the classical Parkinson's disease symptoms," said Michael McDonald, CMO at Oxford
BioMedica plc. "There's great need, a clear hypothesis and we know the target organ. Where people struggle is, if you take cells, to what extent do you need to distribute them in the striatum?"

In December 2007, Oxford BioMedica started a Phase I/II trial of its ProSavin, a lentivector carrying three genes encoding enzymes for dopamine synthesis. The gene therapy will be administered via MRI-guided intrastriatal stereotaxic injection in 6 patients, and an additional 12 patients will be enrolled in a second stage of the trial to confirm the efficacy of the optimal dose.

"With gene-therapy vectors, some of the issues are frankly not that different" from those faced by stem cell therapies, said McDonald. "But we have things working for us that might tip the favor for viral vectors-they're physically much smaller and might thus better spread through diffusion."

Other disease-modifying gene therapies in the clinic for PD include CERE-120 from Ceregene Inc. and partner Genzyme Corp. The adeno-associated viral type 2 vector encodes the gene for neurturin, an analog of glial cell line-derived neurotrophic factor (GDNF). It is in Phase II testing. ${ }^{2}$

McDonald told SciBX he would like to see the Korean group's hESCderived neurons tested in larger animals. Because distribution in the striatum is a key issue, he said, "the size of the animal really matters. As you go from rodent to nonhuman primate to man, you're increasing the volume of the striatum by an order of 6-10-fold."

Avinoam Kadouri, acting CTO at BrainStorm Cell Therapeutics Inc., said he wanted to see longer-term animal data on the hESCderived neurons. "Will the cells just sit there and produce dopamine-if so, for how long? That needs to be clarified," he said.

Another issue with the neurons is "how you control the amount of dopamine they're secret-

ing," said Kadouri. "This is why we moved away from dopamine-producing cells to neurotrophic cells."

Too much dopamine in the brain can cause neurological side effects including dyskinesia and dystonia.

BrainStorm harvests a patient's bone marrow and isolates the mesenchymal stem cells. Those cells are differentiated in serum-free media into cells that secrete neurotrophic factors such as nerve growth factor and GDNF. In a rodent model of PD, the cells remained viable for three months post-transplant and regenerated dopaminergic cells and nerve axons. Rodents receiving the cells had improved motor function compared with untreated controls.

Although neurotrophic factors have not enjoyed much, if any, success in the clinic, Kadouri noted that "the situation is different here. We're not giving a bolus of just the growth factor. We're giving cells that secrete a family of factors."

Kadouri also noted that although the hESC-derived neurons in the PNAS paper did not form teratomas, the Korean group "was able to differentiate the cells and keep them dividing. To me, that's a red light-when a cell differentiates, it should stop growing." 


\section{TARGETS \& MECHANISMS}

Marc Rubin, president and CEO of Titan Pharmaceuticals Inc., also thinks it is important to treat PD with cells that do not divide. Instead of embryonic stem cells, Titan is using human retinal pigmented epithelial cells.

"They sit there in the retina, they don't divide and constitutively make dopamine," he said. "So we asked 'what if we place them in the brain-would they sit there as fully functional dopamine microfactories in the brain?"'

In a pilot study in six patients, Titan and partner Bayer Schering Pharma AG, a subsidiary of Bayer AG, showed that the biotech's Spheramine retinal pigmented epithelial cells produced average motor function improvements of $41-48 \%$ at 2 years, depending on whether patients were off or on other PD medications. A 71-patient Phase IIb trial is ongoing, and data are expected this year.

\section{REFERENCES}

1. Cho, M.S. et al. Proc. Natl. Acad. Sci. USA; published online Feb. 27, 2008; doi:10.1073/pnas.0712359105

Contact: Dong-Wook Kim, Stem Cell Research Center, Seoul, South Korea

e-mail: dwkim2@yuhs.ac

2. Mikulski, M. BioCentury 15(28), A9; June 25, 2007

COMPANIES AND INSTITUTIONS MENTIONED

Bayer AG (FSE:BAY), Leverkusen, Germany

Bayer Schering Pharma AG, Berlin, Germany

BrainStorm Cell Therapeutics Inc. (OTCBB:BCLI), New York, N.Y

Ceregene Inc. San Diego, Calif.

Genzyme Corp. (NASDAQ:GENZ), Cambridge, Mass.

Oxford BioMedica plc (LSE:OXB), Oxford, U.K.

Stem Cell Research Center, Seoul, South Korea

Titan Pharmaceuticals Inc. (AMEX:TTP), South San Francisco, Calif. 